\title{
First Result of NEOS Experiment
}

\author{
Kim Siyeon* \\ Affiliation:Chung-Ang University \\ On behalf of NEOS Collaboration \\ E-mail: siyeonecau.ac.kr
}

\begin{abstract}
Neutrino Experiment for Oscillation at Short baseline (NEOS) targeted the search of singlet neutrinos as natural extension to Standard Model three neutrinos. The electron antineutrinos emerging from a reactor of 2.8-GW thermal power were detected in tendon gallery, 24 meters away from the reactor core. The experiment was performed at Hanbit Nuclear Power Plant in YoungGwang, Korea for eight months. The baseline is optimized for the oscillation with mass-squared difference about $1 \mathrm{eV}^{2}$. A ton of Gd-loaded liquid scintillator collected 1976 events per day with signal-to-background rate 22 during reactor-on period. The shape analysis exhibits a moderate spectrum consistent to those obtained in other reactor neutrino oscillation for about $1.5-\mathrm{km}$ distance. The 5-MeV excess also appears in the spectrum shape of NEOS as seen commonly in Daya Bay, RENO and Double Chooz. Although our analysis drew an exclusion curve of high sensitivity, a singlet neutrino state has not been verified for a scale between the mass of atmospheric neutrino and the order of eV. However, the result of NEOS presents the direction to further search of sterile neutrinos. Possible strategies using the result and the prospect of future sterile neutrino search will be discussed.
\end{abstract}

35th International Cosmic Ray Conference - ICRC2017

10-20 July, 2017

Bexco, Busan, Korea

${ }^{*}$ Speaker. 


\section{Introduction}

The mixing between three neutrino mass eigenstates and weak interaction eigenstates was experimentally confirmed and the mass-squared differences and three mixing angles have been updated from the atmospheric, reactor, and accelerator neutrino oscillation experiments for the last decades [1] [2]. Mass hierarchy, the mass of the lightest neutrino, Majorana nature, and CP phase in three-neutrino frame are ongoing issues to be determined in future experiments [3][4]. Even though the number of active light neutrinos are limited to three from the $Z$ boson decay measurements [5], the existence of extra neutrinos has not been ruled out, which are not involved in the Standard Model weak interaction. That is why they are called sterile neutrinos [6]. Rather, there a various models of sterile neutrinos in order to explain the lightness of neutrino masses, the origin of baryon asymmetry, and etc.

The existence of sterile neutrinos can be identified by the active-sterile neutrino oscillations. Indeed, LSND experiment[7] for $\bar{v}_{\mu} \rightarrow \bar{v}_{e}$ transition reported an observation of oscillation with mass-squared difference larger than $0.01 \mathrm{eV}^{2}$ implying a sterile neutrino. On the other hand, MiniBooNE experiment[8] was not conclusive in confirming the LSND result. There are also two types of known anomalies in $v_{e}$ disappearance, Gallium anomaly and Reactor anomaly. Gallium radioactive source experiments GALLEX and SAGE[9] reported the disappearance of $v_{e} \mathrm{~s}$ at a few meter baseline and its measured-to-predicted ratio is $0.88 \pm 0.05$. The other type of anomaly was revealed in dozens of short baseline reactor neutrino experiments performed whose baselines are between 8 and 100 meters, e.g., Bugey[10]. All those anomalies indicate the order of $1 \mathrm{eV}$ as a most probable mass scale of sterile neutrino in the oscillation with active neutrinos. The reactor antineutrino anomaly became an issue because the reactor neutrino event rates showed about $6 \%$ deficit in comparison to the updated theoretical expectations [11,12] before the Neutrino Experiment for Oscillation at Short baseline (NEOS experiment).

Former reactor experiments, Daya Bay [13], RENO [14], and Double Chooz [15], also consistently obtained the same deficit in the absolute flux at near detectors, although their main target was measuring $\theta_{13}$. Reactor antineutrino oscillation turns out to play rather pivotal role in the prospect of neutrino physics and other related fields. The reactor experiments with multiple detectors commonly reported a 5-MeV excess in spectrum [13][14][15]. Both the excess and the anomalous deficit require better understanding in both neutrinos and nuclear processes in reactors $[12,16,17]$. If the existence of light sterile neutrinos is confirmed in experiment, the number of neutrinos and mass bound in Cosmology, as well as the search of dark matter candidate, can establish a framework to approach their solutions based on the concrete fact. A number of reactor experiments are being launched or taking data to search a sterile neutrino in short-baseline oscillation [18][19][20][21]. There is a variety of baselines within the experiments, and each baseline intends a different value of sterile neutrino mass. It is expected that the reactor experiments will draw some conclusion on the light sterile neutrinos. Neutrino Experiment for Oscillation at Short baseline(NEOS) has a unique 24-meter baseline among those experiments. In this presentation, we will share the design of detector, the spectral shape analysis, and the exclusion curves obtained from observation. 


\section{Experiment Setup}

The experimental site of NEOS is Hanbit Nuclear Power Plant (NPP) in Younggwang, Korea, which consists of six commercial reactors. The Reactor Unit 5 in Hanbit NPP with $2.8 \mathrm{GW}_{\text {th }}$ thermal power was selected as the source of electron antineutrinos. The reactor core is cylindrical shape of $3.1 \mathrm{~m}$ diameter and $3.8 \mathrm{~m}$ height. The fuel is Low Enriched Uranium (LEU) with $4.6 \%$ ${ }^{235} \mathrm{U}$. NEOS detector is placed in Tendon Gallery of the reactor, aiming the search of disappearance of electron antineutrinos from the oscillation over 24-m baseline. The gallery is an underground hall below the reactor, of which overburden is 20 meter water equivalent(m.w.e).

NEOS detector consists of active target, buffer tank where photo-multiplier tubes(PMT) installed, shields, muon detectors, and Data AQuisition (DAQ) system as shown in Figure. 1. The active target material is a liquid scintillator and is contained in a cylindrical stainless steel tank whose inside is covered by PTFE reflector. The material is homogeneous in a single 1000-L container, and is a 9:1 mixture of Linear Alkyl Benzene (LAB) and Di Isopropyl Naphthalene (DIN)[22]. The mixture of the recipe turns out to be better than LAB-only liquid scintillator in improving the pulse shape discrimination (PSD)[23]. In addition, $0.5 \%$ Gadolinium (Gd) is doped into the scintillator for neutron capture on $\mathrm{Gd}$, so that we could obtain separate signals of the positron and the neutron produced from inverse beta decay(IBD), $\bar{v}_{e}+p \rightarrow e^{+}+n$. The combination of the two signals is called an IBD candidate.

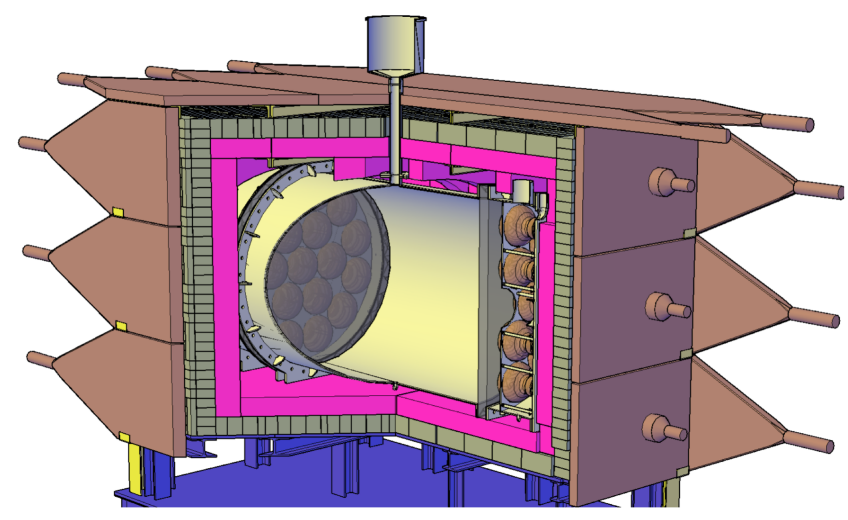

Figure 1: NEOS detector. It consists of active target, buffer tanks with PMTs, shields, and muon detector.

There are two buffer tanks filled with mineral oil at both sides of the target and two acrylic windows between the target and the buffers. Nineteen R5912 (8-inch) PMTs are installed in each buffer tank. There are also two kinds of shields; one is $10-\mathrm{cm}$ thick borated polyethylene against neutrons and the other is $10-\mathrm{cm}$ thick lead against external gammas. Muon detectors for muon veto consists fifteen plates of plastic scintillator with PMTs attached, which cover the surface of the detector except the bottom side.

Data was taken from August 2015 until May 2016 and the reactor-on and -off period are about 180 and 46 days, respectively. The number of IBD candidates per day is 2000 and 80 during the operating period and off period, respectively. The on-to-off ratio of candidate numbers is about 24. The energy resolution is about $5 \%$ at $1 \mathrm{MeV}$, which is the most probable range for light sterile neutrinos, according to the prediction of reactor anomaly interpretation. 


\section{Data Analysis}

Only the events with energy above $0.6 \mathrm{MeV}$ are selected as single events and IBD reconstructions are made with those single events. Figure. 2 shows the energy distribution of single events. An IBD candidate consists of a signal by a positron and its annihilation and a signal by a neutron capture on Gd. They are called the prompt signal (S1) and the delayed signal (S2), respectively. The energy of S1 is given by a simple re-estimation the energy of a detected neutrino, and it ranges 1-10 MeV. Total gamma energy due to $\mathrm{n}-\mathrm{Gd}$ capture is about $8 \mathrm{MeV}$, but the energy of S2 ranges 4-10 MeV because some of gammas escape from the target. The size is not large enough to collect all the gammas in it. The time difference between two separated signals is 1-30 $\mu$ s since the neutron capture takes some time compared to S1.

In addition, there are criteria applied for reducing the backgrounds. The pulse shape discrimination is used against fast neutrons [24], and the multiplicity cut is used against multiple neutrons as in spallation. The PSD cut is set to accept $99.9 \%$ of gamma-like events, as shown in Figure. 3). The multiplicity cut should not allow either a single event between S1 and S2, a single event within $30 \mu$ s before $\mathrm{S} 1$ or another single event other than $\mathrm{S} 2$ within $150 \mu \mathrm{s}$ after $\mathrm{S} 1$. The time window for muon veto is $150 \mu \mathrm{s}$.

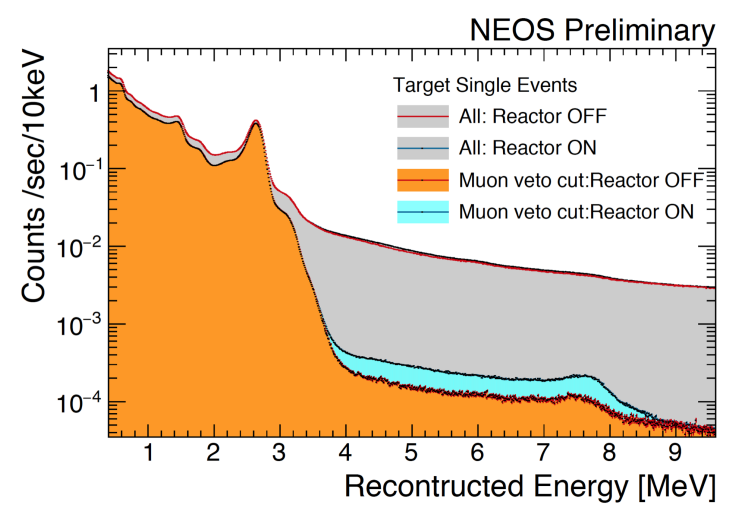

Figure 2: Energy spectrum of selected single events. The gray-filled area indicates the spectrum before muon veto, while the orange- and cyan-filled regions indicate the spectrum after muon veto. The blue and red lines denote the reactor-on and -off period, respectively.

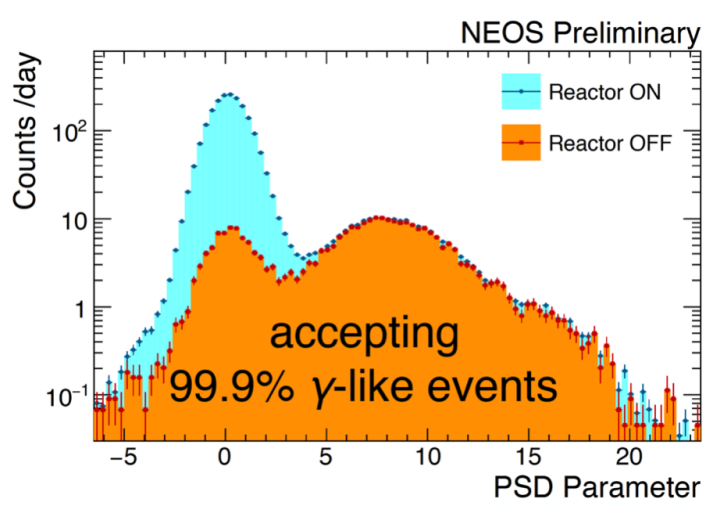

Figure 3: Pulse shape distribution. The orangefilled area is the data during reactor-off period and the cyan-filled area is the data during reactor-on period. The cut is selected so as to accept $99.9 \%$ gamma-like events.

The data in the reactor-on period has both neutrino signals and backgrounds. On the other hand, the data of the reactor-off period has only the backgrounds. The ratio of signal to background is about 22, and the energy spectrum of prompt signal is given in the top plot of Figure.4. Data analysis refers Huber and Mueller's model[12] with Vogel's IBD cross section [25] as expectation. The spectrum excess around $5 \mathrm{MeV}$ appears clearly when the data is in comparison with $\mathrm{HM}$ model. In order to avoid the conflict from the excess, our analysis takes the strategy referring Daya Bay spectrum[26] obtained in their near detector as the expected spectrum. That is, the aspect of oscillation due to the mass of fourth neutrino has been looked into by comparing with Daya Bay 


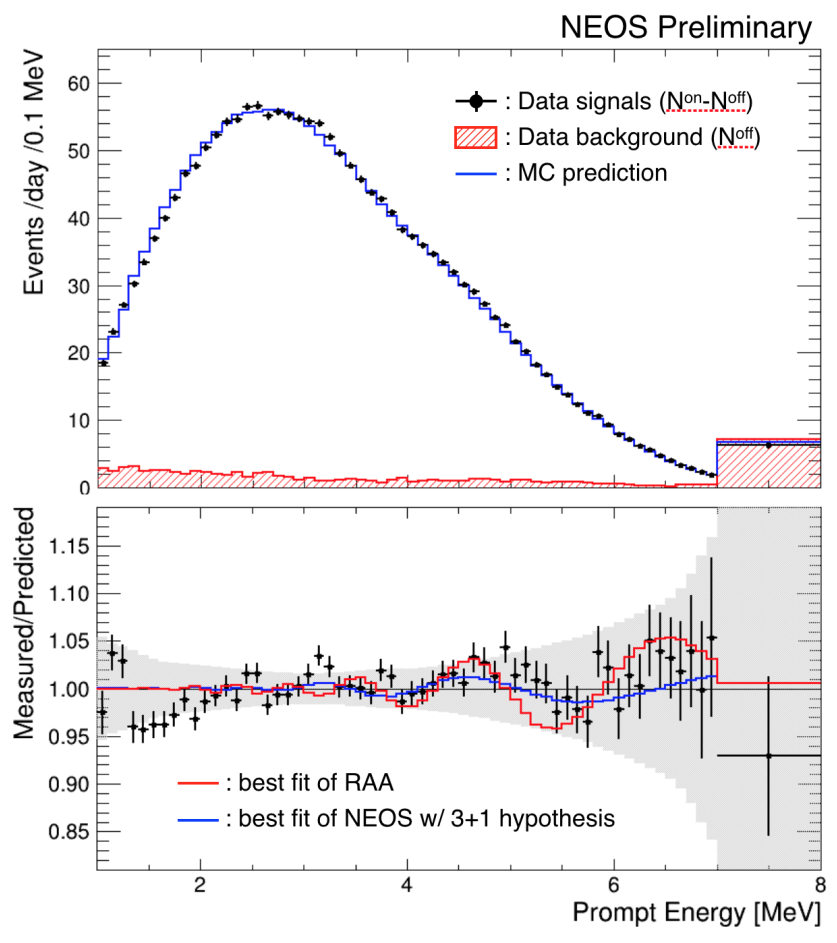

Figure 4: (top) Prompt energy spectrum of NEOS. Black dots with error bars represent the data corresponding to signals, i.e., data of reactor on subtracted by data of reactor off, and red-filled area indicates the data of background while the reactor is off. The blue line is MC prediction referring Daya Bay absolute spectrum. The last bin is a sum of 7 to $10 \mathrm{MeV}$. (bottom) The ratio of measured to predicted. The red line is the case of the best fit of RAA, while the blue line is the case of the best fit of NEOS with $3+1$ hypothesis.

spectrum. Any possible effects due to the different fission fractions between Daya Bay and NEOS was appropriately modified.

\section{Results and Remarks}

The first oscillation analysis of NEOS results is shape-only analysis [27]. The chi-square is estimated by the following expression,

$$
\chi^{2}=\sum_{i, j}\left(N_{i}^{\mathrm{on}}-\left(t_{\mathrm{on}} / t_{\mathrm{off}}\right) N_{i}^{\mathrm{off}}\right) M_{i j}^{-1}\left(N_{j}^{\mathrm{on}}-\left(t_{\mathrm{on}} / t_{\mathrm{off}}\right) N_{j}^{\mathrm{off}}-N_{j}^{\mathrm{exp}}\right)
$$

where $N^{\text {on }}$ and $N^{\text {off }}$ are the measured event numbers during the reactor-on and -off periods, respectively. The $N^{\text {exp }}$ denotes the predicted event number, and $M_{i j}$ is the element of covariance matrix including both statistical and systematic uncertainties. The $t_{\mathrm{on}}$ and $t_{\text {off }}$ denote the live times for reactor-on and -off data, respectively. Within the $3+1$ hypothesis, the bottom plot of Figure. 4 shows the best fits of NEOS and RAA. As a result of significance test, no strong evidence of sterile neutrino was found. The raster scan gives rise to an exclusion curve in $\sin ^{2} 2 \theta_{14}-\Delta m_{41}^{2}$ space at $90 \% \mathrm{CL}$ as shown in Figure. 5. For the range $0.2 \mathrm{eV}^{2}<\Delta \mathrm{m}_{41}^{2}<2.3 \mathrm{eV}^{2}$, the upper limit of $\sin ^{2} 2 \theta_{14}$ is restricted to be less than 0.1 . The curve excludes a proposed region for a sterile neutrino.

NEOS experiment has additional outcomes besides the sterile neutrino search. The detector monitored the ramping-up steps of turning-on process of a reactor. It is the first single-reactor experiment that confirmed the $5-\mathrm{MeV}$ excess in the reactor neutrino spectrum. Further search of sterile neutrinos has a few ongoing directions; one is the measurement of $\theta_{24}$ from appearance experiments of accelerator neutrino oscillation, and another is very-short-baseline oscillation of heav- 


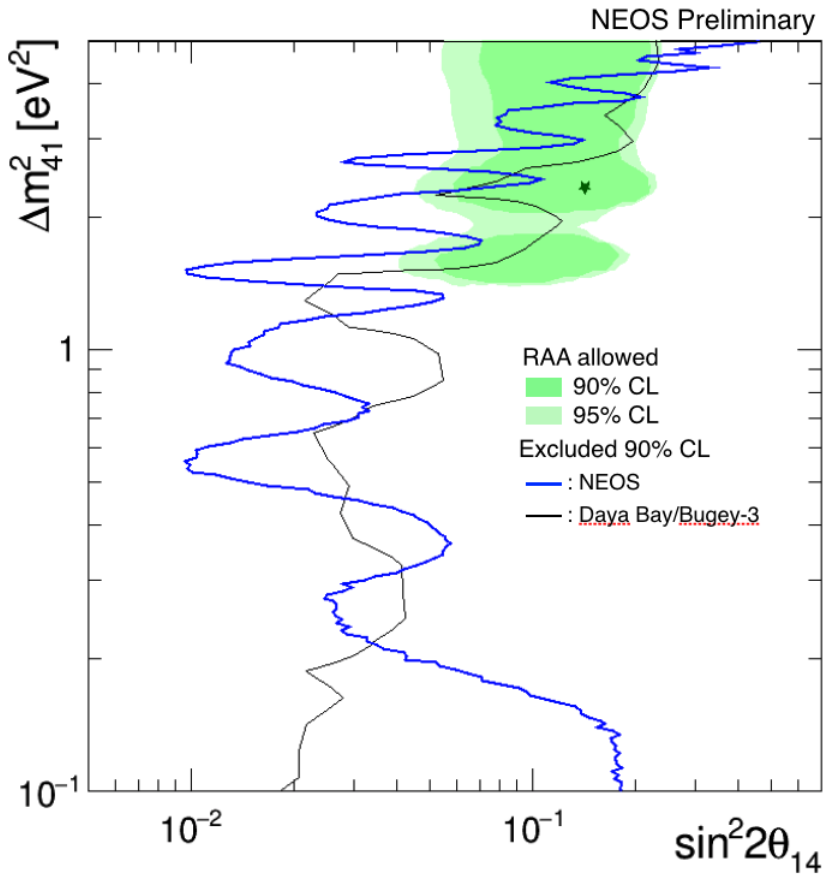

Figure 5: Excluded region of NEOS (blue line). Black thin line is the combined result of Daya Bay and Bugey-3[?]. The green-filled regions are the allowed areas with $90 \%$ (dark green) and 95\% (light green) CL. in RAA

ier sterile neutrinos. A number of experiments mentioned in Introduction have already launched, expecting their results in near future. NEOS has also proposed its second phase in an improved condition to search sterile neutrinos.

\section{References}

[1] G. L. Fogli, E. Lisi, A. Marrone, D. Montanino, A. Palazzo and A. M. Rotunno, Global analysis of neutrino masses, mixings and phases: entering the era of leptonic CP violation searches, Phys. Rev. D 86, 013012 (2012)

[2] K. A. Olive et al. [Particle Data Group Collaboration], Review of Particle Physics, Chin. Phys. C 38 (2014) 090001.

[3] M. C. Gonzalez-Garcia, M. Maltoni and T. Schwetz, Updated fit to three neutrino mixing: status of leptonic CP violation, JHEP 1411, 052 (2014)

[4] M. Mezzetto, Experimental Outlook, Neutrino 2016, XXVII International conference on neutrino physics and astrophysics, 4-9 July 2016

[5] S. Schael et al. [ALEPH and DELPHI and L3 and OPAL and SLD Collaborations and LEP Electroweak Working Group and SLD Electroweak Group and SLD Heavy Flavour Group], Precision electroweak measurements on the $Z$ resonance, Phys. Rept. 427, 257 (2006) doi:10.1016/j.physrep.2005.12.006

[6] M. Archidiacono, N. Fornengo, C. Giunti, S. Hannestad and A. Melchiorri, Sterile neutrinos: Cosmology versus short-baseline experiments, Phys. Rev. D 87, no. 12, 125034 (2013)

[7] C. Athanassopoulos et al. [LSND Collaboration], Evidence for anti-muon-neutrino $\longrightarrow$ anti-electron-neutrino oscillations from the LSND experiment at LAMPF, Phys. Rev. Lett. 77, 3082 (1996) 
[8] A. A. Aguilar-Arevalo et al. [MiniBooNE Collaboration], Improved Search for $\bar{v}_{\mu} \rightarrow \bar{v}_{e}$ Oscillations in the MiniBooNE Experiment, Phys. Rev. Lett. 110, 161801 (2013)

[9] J. N. Abdurashitov et al., Measurement of the response of a Ga solar neutrino experiment to neutrinos from an Ar-37 source, Phys. Rev. C 73, 045805 (2006)

[10] Y. Declais et al., Search for neutrino oscillations at 15-meters, 40-meters, and 95-meters from a nuclear power reactor at Bugey, Nucl. Phys. B 434, 503 (1995).

[11] G. Mention, M. Fechner, T. Lasserre, T. A. Mueller, D. Lhuillier, M. Cribier and A. Letourneau, The Reactor Antineutrino Anomaly, Phys. Rev. D 83, 073006 (2011)

[12] P. Huber, On the determination of anti-neutrino spectra from nuclear reactors, Phys. Rev. C 84, 024617 (2011) Erratum: [Phys. Rev. C 85, 029901 (2012)]

[13] F. P. An et al. [Daya Bay Collaboration], Measurement of the Reactor Antineutrino Flux and Spectrum at Daya Bay, Phys. Rev. Lett. 116, no. 6, 061801 (2016) Erratum: [Phys. Rev. Lett. 118, no. 9, 099902 (2017)]

[14] J. H. Choi et al. [RENO Collaboration], Observation of Energy and Baseline Dependent Reactor Antineutrino Disappearance in the RENO Experiment, Phys. Rev. Lett. 116, no. 21, 211801 (2016)

[15] Y. Abe et al. [Double Chooz Collaboration], Indication of Reactor $\bar{v}_{e}$ Disappearance in the Double Chooz Experiment, Phys. Rev. Lett. 108, 131801 (2012)

[16] D. A. Dwyer and T. J. Langford, Spectral Structure of Electron Antineutrinos from Nuclear Reactors,” Phys. Rev. Lett. 114, no. 1, 012502 (2015)

[17] A. C. Hayes, J. L. Friar, G. T. Garvey, G. Jungman and G. Jonkmans, Systematic Uncertainties in the Analysis of the Reactor Neutrino Anomaly, Phys. Rev. Lett. 112, 202501 (2014)

[18] M. Pequignot [Nucifer and Stereo Collaborations], The Nucifer and Stereo reactor antineutrino experiments, Nucl. Part. Phys. Proc. 265-266, 126 (2015).

[19] M. Danilov [DANSS Collaboration], Sensitivity of the DANSS detector to short range neutrino oscillations, PoS EPS -HEP2013, 493 (2013)

[20] N. Ryder [SoLid Collaboration], First results of the deployment of a SoLid detector module at the SCK-CEN BR2 reactor, PoS EPS -HEP2015, 071 (2015)

[21] J. Ashenfelter et al. [PROSPECT Collaboration], The PROSPECT Physics Program, arXiv:1512.02202 [physics.ins-det].

[22] B. R. Kim et al., Development and Mass Production of a Mixture of LAB-and DIN-based Gadolinium-loaded Liquid Scintillator for the NEOS Short-baseline Neutrino Experiment, arXiv:1511.05551 [physics.ins-det].

[23] B. R. Kim et al., Pulse shape discrimination capability of metal-loaded organic liquid scintillators for a short-baseline reactor neutrino experiment, Phys. Scripta 90, no. 5, 055302 (2015).

[24] Y. J. Ko et al., Comparison of fast neutron rates for the NEOS experiment, J. Korean Phys. Soc. 69, no. 11, 1651 (2016)

[25] P. Vogel and J. F. Beacom, Angular distribution of neutron inverse beta decay, anti-neutrino(e) $+p$ $\longrightarrow$ e+ + n, Phys. Rev. D 60, 053003 (1999)

[26] F. P. An et al. [Daya Bay Collaboration], Improved Measurement of the Reactor Antineutrino Flux and Spectrum at Daya Bay, Chin. Phys. C 41, no. 1, 013002 (2017)

[27] Y. J. Ko et al., Sterile Neutrino Search at the NEOS Experiment, Phys. Rev. Lett. 118, no. 12, 121802 (2017) 